\title{
A COMMUTATIVE BANACH ALGEBRA WITH FACTORIZATION OF ELEMENTS BUT NOT OF PAIRS
}

\author{
S. I. OUZOMGI
}

(Communicated by Palle E. T. Jorgensen)

\begin{abstract}
We find a one-point Gleason part $\phi$ off the Šilov boundary of $H^{\infty}(\Delta)$ such that the maximal ideal $M_{\phi}$ factors but such that pairs do not factor in $M_{\phi}$.
\end{abstract}

\section{INTRODUCTION}

A topological algebra $A$ factors if, for each $y \in A$, there exist $a, x \in A$ with $y=a x$. We say that pairs factor in $A$ if, for each $y_{1}, y_{2} \in A$ there exist $a \in A$ and $x_{1}, x_{2} \in A$ with $y_{1}=a x_{1}$ and $y_{2}=a x_{2}$. Also, $A$ strongly factors if, for each sequence $\left(y_{n}\right)$ tending to zero, there exist $a \in A$ and a sequence $\left(x_{n}\right)$ tending to zero with $y_{n}=a x_{n}$. A bounded approximate identity for a Banach algebra $A$ is a net $\left(e_{\alpha}\right) \subset A$ such that $x e_{\alpha} \rightarrow x$ and $e_{\alpha} x \rightarrow x$ for each $x \in A$ and such that $\sup \left\|e_{\alpha}\right\|<\infty$. It is well known that if $A$ has a bounded approximate identity, then it strongly factors and hence factors $[1, \mathrm{p}$. 62]. But a commutative Banach algebra may factor even if it does not have a bounded approximate identity: a nonseparable example is given in [4, p. 166] and it is easy to see that this example strongly factors. More recently, Willis gave an example of a commutative, separable Banach algebra which strongly factors, but has no bounded approximate identity, [10].

In this paper, we give an example of a commutative, nonseparable Banach algebra which factors, but does not strongly factor. More precisely, our algebra contains two elements with no common factor.

\section{Preliminaries}

Throughout, $X$ will denote a compact Hausdorff space and $C(X)$ the algebra of all continuous functions on $X$ with the uniform norm

$$
\|f\|_{X}=\sup \{|f(x)|: x \in X\} .
$$

Also, if $S \subset X$, we let $\|f\|_{S}=\sup \{|f(x)|: x \in S\}$.

Received by the editors April 23, 1990.

1980 Mathematics Subject Classification (1985 Revision). Primary 46J10. 
Definition 2.1. A uniform algebra on $X$ is a closed subalgebra of $C(X)$ that contains the constants and separates points of $X$.

Let $A$ be a uniform algebra. For $x \in X$, we shall denote by $M_{x}$ the maximal ideal $M_{x}=\{f \in A: f(x)=0\}$ that consists of functions in $A$ vanishing at $x$.

Definition 2.2. A boundary for a uniform algebra $A$ is a subset $S$ of $X$ on which the modulus of every function in $A$ assumes its maximum.

Among all closed boundaries for $A$ there is a smallest one $[9,7.4]$ which is called the Šilov boundary.

Definition 2.3. A point $x \in X$ is called a peak point for $A$ if there exists $f \in A$ such that $f(x)=\|f\|_{X}=1$ and $|f(y)|<1$ for $y \neq x$. It is called a strong boundary point for $A$ if for each neighborhood $U$ of $x$ there exists $f \in A$ such that $f(x)=\|f\|_{X}=1$ and $|f(y)|<1$ for $y \in X \backslash U$.

It is easily seen that a peak point is a strong boundary point and a strong boundary point necessarily belongs to the Šilov boundary. Also, if $X$ is metrizable, then $C(X)$ is separable and so is every closed subalgebra of $C(X)$. In this case, peak points and strong boundary points coincide. The following theorem relates maximal ideals and strong boundary points for uniform algebras.

Theorem 2.4. The maximal ideal $M_{x}$ of a uniform algebra $A$ has a bounded approximate identity if and only if $x$ is a strong boundary point.

Proof. See [2, p. 101] and [3, 5.8].

\section{Parts AND MAXimal ideals in $H^{\infty}(\Delta)$}

Let $\Delta$ be the open unit disc in the complex plane and let $H^{\infty}(\Delta)$ be the algebra of bounded analytic functions on $\Delta$. Under the norm

$$
\|f\|=\sup \{|f(z)|:|z|<1\},
$$

$H^{\infty}(\Delta)$ is a commutative Banach algebra with identity. Let $\Phi$ be the maximal ideal space of $H^{\infty}(\Delta)$ and consider the Gelfand map $f \mapsto \hat{f}$ from $H^{\infty}(\Delta)$ to $C(\Phi)$ defined by $\hat{f}(\phi)=\phi(f)$ for $\phi \in \Phi$. It is not hard to see that this is an isometry and therefore $H^{\infty}(\Delta)$ is isometrically isomorphic to its image $\widehat{H}^{\infty}(\Delta)$, a uniformly closed subalgebra of $C(\Phi)$ that separates points of $\phi$ and contains the constants. Hence $H^{\infty}(\Delta)$ can be regarded as a uniform algebra on the compact space $\Phi$.

For $\phi$ and $\psi$ in $\Phi$, we consider the pseudohyperbolic metric

$$
\rho(\phi, \psi)=\sup \{|\hat{f}(\phi)|:\|f\| \leq 1 \text { and } \hat{f}(\psi)=0\},
$$

and the relation $\phi \sim \psi$ if and only if $\rho(\phi, \psi)<1$. This defines an equivalence relation [5, p. 142].

Definition 3.1. The equivalence classes of $\Phi$ under the equivalence relation $\sim$ are called Gleason parts or simply parts for $H^{\infty}(\Delta)$. 
Definition 3.2. An analytic disc is the image of a one-to-one continuous map $L$ from $\Delta$ to $\Phi$ such that $\hat{f} \circ L$ is analytic for each $f \in H^{\infty}(\Delta)$.

It is easily seen that the evaluation maps on $\Delta$ form an analytic disc.

Gleason parts for the algebra $H^{\infty}(\Delta)$ have been studied by Hoffman [8] where the next theorem is proved. See also $[9,17.1]$.

Theorem 3.3. Each part for the algebra $H^{\infty}(\Delta)$ is either a one-point part or an analytic disc.

Theorem 3.4. If $f$ is a bounded analytic function on $\Delta$ and if $\alpha_{1}, \alpha_{2}, \alpha_{3}, \ldots$ are the zeros of $f$, each repeated as often as its multiplicity, then

$$
\sum_{n=1}^{\infty}\left(1-\left|\alpha_{n}\right|\right)<\infty
$$

Also, if $\left(\alpha_{n}\right)$ is a sequence in $\Delta$ satisfying (2) and such that $\alpha_{n} \neq 0$ for all $n$, if $k$ is a nonnegative integer, and if

$$
B(z)=z^{k} \prod_{n=1}^{\infty}\left(\frac{\left|\alpha_{n}\right|}{\alpha_{n}} \frac{\alpha_{n}-z}{1-\bar{\alpha}_{n} z}\right) \quad(|z|<1)
$$

then $B \in H^{\infty}(\Delta)$ and $B$ has no zeros except at the points $\alpha_{n}$, and at the origin if $k>0$. See [7, p. 63].

The function $B$ of the theorem is called a Blaschke product.

Definition 3.5. An inner function $f(z)$ is a bounded analytic function such that $\left|f\left(e^{i \theta}\right)\right|=1$ almost everywhere on the unit circle.

Theorem 3.6 [7, p. 66]. Let $f$ be a nonzero function in $H^{\infty}(\Delta)$. Then $f$ is uniquely expressible in the form $f=B g$, where $B$ is a Blaschke product and $g$ a bounded analytic function without zeros.

Theorem 3.7 [6, p. 194]. Let $\phi$ be a complex homomorphism on $H^{\infty}(\Delta)$. Then the following are equivalent:

(i) $\phi$ lies in the Silov boundary for $H^{\infty}(\Delta)$; .

(ii) $|\phi(B)|>0$ for every Blaschke product $B$;

(iii) $|\phi(u)|=1$ for every inner function $u$.

For lack of a suitable reference, we include a proof of the following wellknown result (see [5, p. 162, Exercise 3], and [6, p. 438]). We will need the function $f$ of the proof for our example.

Theorem 3.8. There exists a one-point part $\phi$ off the Silov boundary of $H^{\infty}(\Delta)$ and a function $f \in H^{\infty}(\Delta)$ such that $f$ has no zeros in $\Delta$ and such that $f \in M_{\phi}$. Proof. We consider the function defined by

$$
f(z)=\exp \left(\frac{z+1}{z-1}\right) \quad(|z|<1) .
$$


Clearly $|f(z)|<1$ for $z$ in $\Delta$. Also, if we look at the boundary behavior of $f$ we see that $|f(z)|=1$ for all $z \neq 1$ on the unit circle and this leads us to say that $\|f\|=1$. Let $Z(\hat{f})=\{\phi \in \Phi: \hat{f}(\phi)=0\}$. Since $f(z)$ tends to zero as $z$ tends to 1 along the real axis, $\hat{f}$ has no inverse and so $Z(\hat{f})$ is nonempty. Furthermore, since $f(z)$ is an inner function and $\phi(f)=0$, then by Theorem 3.7 we have $Z(\hat{f}) \cap \Gamma=\varnothing$, where $\Gamma$ is the Šilov boundary of $H^{\infty}(\Delta)$.

We next show that $Z(\hat{f})$ is a union of parts. Let $\phi \in Z(\hat{f})$ and let $\theta$ be in the same Gleason part as $\phi$ with $\theta(f) \neq 0$. Then, if $f_{n}$ is the $n$th root of $f$, which clearly belongs to $H^{\infty}(\Delta)$, we have

$$
\rho(\phi, \theta) \geq \sup _{n}\left|f_{n}(\theta)\right|=\sup _{n}|\hat{f}(\theta)|^{1 / n}=\lim _{n}|\hat{f}(\theta)|^{1 / n}=1 .
$$

This is a contradiction because $\phi$ and $\theta$ lie in the same part. This shows that $\hat{f}=0$ on the full Gleason part containing $\phi$. So for each $\phi \in Z(\hat{f})$, the Gleason part containing $\phi$ is contained in $Z(\hat{f})$. Thus $Z(\hat{f})$ is a union of parts.

To complete the proof of the theorem, we suppose that $Z(\hat{f})$ does not contain a one-point part, and so by Theorem 3.3 is a union of analytic discs, and we seek a contradiction.

Let $\mathscr{F}$ be the family of nonempty, closed subsets of $Z(\hat{f})$ which are a union of analytic discs. This family is nonempty because $Z(\hat{f})$ is already one of its elements. Let $\left\{F_{\lambda}: \lambda \in \Lambda\right\}$ be a decreasing chain in $\mathscr{F}$ and consider $G=\bigcap_{\lambda \in \Lambda} F_{\lambda}$. Clearly $G$ is nonempty because it is the intersection of closed subsets which have the finite intersection property in a compact space. Also, if $\phi \in G$, then $\phi \in F_{\lambda}$ and the part containing $\phi$ is included in $F_{\lambda}$ for each $\lambda$ because $F_{\lambda}$ is a union of parts. This shows that $G$ is an element of the family $\mathscr{F}$, and so Zorn's lemma asserts the existence of a minimal element $F_{0}$ in $\mathscr{F}$.

Since $F_{0}$ is not a singleton, and since $H^{\infty}(\Delta)$ separates points of $\Phi$, there exists a function $g$ in $H^{\infty}(\Delta)$ such that $g$ is not constant on $F_{0}$. We can suppose that $|g|$ is not constant on $F_{0}$. Let

$$
F_{1}=\left\{\phi \in F_{0}:|\hat{g}(\phi)|=\sup _{\psi \in F_{0}}|\hat{g}(\psi)|\right\} .
$$

Then $F_{1}$ is a nonempty, proper closed subset of $F_{0}$. Let $\phi$ be in $F_{1}$ and let $\Pi$ be the analytic disc to which $\phi$ belongs. Let $L$ be a one-to-one map from the open unit disc onto the Gleason part $\Pi$ with $\hat{g} \circ L$ analytic for all $g \in H^{\infty}(\Delta)$. There exists $z_{0}$ in $\Delta$ such that $L\left(z_{0}\right)=\phi$. Then

$$
\left|\hat{g} \circ L\left(z_{0}\right)\right|=|\hat{g}(\phi)|=\sup _{\psi \in F_{0}}|\hat{g}(\psi)| \geq \sup _{\psi \in \Pi}|\hat{g}(\psi)|=\sup _{z \in \Delta}|\hat{g} \circ L(z)| .
$$

This shows that $\left|\hat{g} \circ L\left(z_{0}\right)\right|=\sup _{z \in \Delta}|\hat{g} \circ L(z)|$ and by the maximum modulus principle we have that $|\hat{g} \circ L|$ is constant on $\Delta$ and $|\hat{g}|$ is constant on $\Pi$ with value $|\hat{g}(\phi)|$. Thus, $\Pi \subset F_{1}$ and since the choice of $\phi$ in $F_{1}$ was arbitrary, $F_{1}$ is a union of parts and $F_{1} \in \mathscr{F}$. This contradicts the minimality of $F_{0}$ and the proof of the theorem is complete. 


\section{EXISTENCE OF A PAIR IN $M_{\phi}$ WITH NO COMMON FACTOR}

Theorem 4.1 [6, p. 412]. Let $\phi$ be an element of $\Phi$. The following are equivalent:

(i) the Gleason part containing $\phi$ is a one-point part;

(ii) if $f \in M_{\phi}$ with $\|f\| \leq 1$, then there exist $f_{1}, f_{2} \in M_{\phi}$ with $f=f_{1} f_{2}$ and $\left\|f_{j}\right\| \leq 1$

Proposition 4.2. Let $\phi$ be a one-point part off the Silov boundary of $H^{\infty}(\Delta)$. Then the maximal ideal $M_{\phi}=\left\{f \in H^{\infty}(\Delta): \phi(f)=0\right\}$ factors, but has no bounded approximate identity.

Proof. Theorem 4.1 shows that $M_{\phi}$ factors. Also, since $\phi$ does not belong to the Šilov boundary, $\phi$ cannot be a strong boundary point. By Theorem 2.4, $M_{\phi}$ has no bounded approximate identity.

We are now ready to show that in the maximal ideal $M_{\phi}$ pairs do not factor and hence $M_{\phi}$ does not strongly factor.

Theorem 4.3. There exists a one-point Gleason part $\phi$ off the Silov boundary of $H^{\infty}(\Delta)$, such that the maximal ideal $M_{\phi}$ factors but pairs do not factor in $M_{\phi}$. Proof. Let $\phi$ and $f$ satisfy the conditions of Theorem 3.8. Theorem 3.7 tells us that there exists a Blaschke product $B_{0}$ in the maximal ideal $M_{\phi}$. To obtain a contradiction, suppose that $B_{0}$ and $f$ have a common factor, that is, there exist $g, h_{1}$, and $h_{2}$ in $M_{\phi}$ with

$$
B_{0}=g h_{1} \text { and } f=g h_{2} .
$$

Since $f$ has no zeros in the open disc, it follows that the zero set of $g$, $Z(g)=\{z \in \Delta: g(z)=0\}$, is empty. We now factor $h_{1}$ as in Theorem 3.6, say $h_{1}=\widetilde{B} \tilde{h}_{1}$, where $\widetilde{B}$ is a Blaschke product and $\tilde{h}_{1}$ is a bounded analytic function without zeros. We have

$$
B_{0}=g \widetilde{B} \tilde{h}_{1}=\widetilde{B}\left(g \tilde{h}_{1}\right) .
$$

Since $Z\left(g \tilde{h}_{1}\right)=Z(g) \cup Z\left(\tilde{h}_{1}\right)=\varnothing$, and since a Blaschke product is uniquely determined by its zeros up to a constant factor, we get $g \tilde{h}_{1}=1$. So $g$ is invertible in $H^{\infty}(\Delta)$. But this is a contradiction because $g \in M_{\phi}$ and an invertible element cannot belong to the maximal ideal $M_{\phi}$.

The maximal ideal $M_{\phi}$ is clearly a nonseparable, commutative Banach algebra. Also since a Banach algebra with a bounded approximate identity strongly factors [1, p. 62], our example does not have a bounded approximate identity. In fact, the results of Dixon $[3,5.10]$ show that it does not have an approximate identity, bounded or unbounded.

The question of whether or not a separable commutative Banach algebra that factors has factorization of pairs or of sequences remains open (see $[3, \S 7])$. 
If $A$ is an algebra and $I$ is an ideal in $A$, we let

$$
I^{2}=\left\{\sum_{i=1}^{n} x_{i} y_{i}: n \in \mathbb{N}, x_{1}, \ldots, x_{n} \in I, y_{1}, \ldots, y_{n} \in I\right\}
$$

and

$$
I^{[2]}=\{x y: x \in I, y \in I\} .
$$

In the recent paper [10], Willis gives a separable, commutative Banach algebra $A$ such that $A^{[2]} \subsetneq A^{2}=A$. We shall next use the one point part off the Šilov boundary of $H^{\infty}(\Delta)$, of Theorem 4.3, to give a nonseparable example of such a commutative Banach algebra.

Theorem 4.4. There exists a nonseparable commutative Banach algebra $A$ such that $A=A^{2}$ but such that $A$ does not factor.

Proof. Let $\phi$ be a one point part off the Šilov boundary of $H^{\infty}(\Delta)$ such that pairs do not factor in the maximal ideal $M_{\phi}$. Let $f$ and $g$ be two elements of $M_{\phi}$ such that $f$ and $g$ have no common factor. Let $A=M_{\phi} \oplus M_{\phi} \oplus M_{\phi}$. With pointwise addition and scalar multiplication and the norm

$$
\|(a, b, c)\|=\|a\|+\|b\|+\|c\| \quad((a, b, c) \in A),
$$

$A$ is a Banach space. Also by defining the product

$$
\left(a_{1}, b_{1}, c_{1}\right)\left(a_{2}, b_{2}, c_{2}\right)=\left(a_{1} a_{2}, a_{1} b_{2}+a_{2} b_{1}, a_{1} c_{2}+a_{2} c_{1}\right)
$$

for $\left(a_{1}, b_{1}, c_{1}\right),\left(a_{2}, b_{2}, c_{2}\right) \in A, A$ becomes a commutative Banach algebra.

Let $(a, b, c) \in A$. Since $M_{\phi}$ factors, there exist $k, l, m, n, p$, and $q \in M_{\phi}$ such that $a=k l, b=m n$ and $c=p q$. Thus

$$
(a, b, c)=(k, 0,0)(l, 0,0)+(m, 0,0)(0, n, 0)+(p, 0,0)(0,0, q)
$$

and so $A=A^{2}$. We claim that the element $(0, f, g)$, where $f$ and $g$ do not have a common factor in $M_{\phi}$, does not factor. Suppose that $(0, f, g)=$ $\left(a_{1}, b_{1}, c_{1}\right)\left(a_{2}, b_{2}, c_{2}\right)$ with $a_{i}, b_{i}, c_{i} \in M_{\phi}$ for $i=1,2$. Then we have

$$
0=a_{1} a_{2}, \quad f=a_{1} b_{2}+a_{2} b_{1} \text { and } g=a_{1} c_{2}+a_{2} c_{1} \text {. }
$$

Since $M_{\phi}$ is an integral domain $0=a_{1} a_{2}$ gives that either $a_{1}=0$ or $a_{2}=0$. It follows that either $f=a_{1} b_{2}$ and $g=a_{1} c_{2}$ or $f=b_{1} a_{2}$ and $g=c_{1} a_{2}$. This is a contradiction because $f$ and $g$ do not have a common factor.

Clearly this example is nonseparable.

\section{REFERENCES}

1. F. F. Bonsall and J. Duncan, Complete normed algebras, Springer-Verlag, New York, 1973.

2. A. Browder, Introduction to function algebras, Benjamin, New York, 1969.

3. P. G. Dixon, Factorization and unbounded approximate identities in Banach algebras, Math. Proc. Cambridge. Philos. Soc. 107 (1990), 557-571.

4. R. S. Doran and J. Wichmann, Approximate identities and factorization in Banach modules, Lecture Notes in Math., vol. 768, Springer-Verlag, 1979. 
5. T. W. Gamelin, Uniform algebras, Prentice-Hall, Englewood Cliffs, NJ, 1969.

6. J. Garnett, Bounded analytic functions, Academic Press, New York, 1981.

7. K. Hoffman, Banach spaces of analytic functions, Prentice-Hall, Englewood Cliffs, NJ, 1969.

8. _ Bounded analytic functions and Gleason parts, Ann. of Math. (2) 86 (1967), 74-111.

9. E. L. Stout, The theory of uniform algebras, Bogden and Quigley, Tarrytown-on-Hudson, New York, 1971.

10. G. A. Willis, Examples of factorization without bounded approximate units, preprint, Research Report no. 12, 1989, Mathematical Centre, Australian National University, Canberra.

Department of Mathematics, The Pennsylvania State University, Ogontz Campus, Abington, Pennsylvania 19001 